\title{
Crystal structure of a new polymorph of $\mathrm{Sr}_{2} \mathrm{TiO}_{4}$ with tetrahedral titanium
}

\author{
D. Pulmannova, C. Besnard, E. Giannini \\ Univeristy of Geneva, Quai Ernest-Ansermet 24, 1205 Geneva, Switzerland \\ dorota.pulmannova@unige.ch
}

$\mathrm{Sr}_{2} \mathrm{TiO}_{4}$, first member of the Ruddlesden-Popper series $\mathrm{Sr}_{n+1} \mathrm{Ti}_{n} \mathrm{O}_{3 n+1}$, has been long known to undergo a phase transition at $1550{ }^{\circ} \mathrm{C}$. This transition makes the growth of single crystals of this material highly challenging, because it usually breaks the crystal into a periodic array of uneven lamellae. While the low temperature tetragonal phase is widely studied due to its close connection to the famous perovskite $\mathrm{SrTiO}_{3}$, there is little information about the high temperature $\alpha$-phase, except for an unindexed powder pattern by Drys\&Trzebiatowski [1].

We stabilized the high-temperature $\alpha-\mathrm{Sr}_{2} \mathrm{TiO}_{4}$ crystals by rapid cooling of the incongruent melt from above the liquidus temperature. The $\alpha$-phase crystallizes in the orthorhombic Pna2 ${ }_{1}$ group with lattice parameters $a=14.2901(5) \AA \mathrm{b}=5.8729(2) \AA \mathrm{c}=10.0872(3) \AA$ and is isostructural to the orthorhombic forms of $\mathrm{Sr}_{2} \mathrm{VO}_{4}$ and $\mathrm{Sr}_{2} \mathrm{CrO}_{4}$ (which belong to the $\beta-\mathrm{K}_{2} \mathrm{SO}_{4}$ structure type). Its structure is formed by a complicated framework of large $\mathrm{SrO}_{x}$ polyhedra with tetrahedral cavities occupied by the transition metal. The tetrahedral coordination of $\mathrm{Ti}^{\mathrm{IV}}$ makes the $\alpha-\mathrm{Sr}_{2} \mathrm{TiO}_{4}$ quite a rare case among titanate compounds, the only other known example being the barium orthotitanate $\mathrm{Ba}_{2} \mathrm{TiO}_{4}[2]$.

However, whereas in $\mathrm{Ba}_{2} \mathrm{TiO}_{4}$ the coordination is tetrahedral in both high- and low-temperature polymorphs and the topotactic relation between the two is known, in the case of $\mathrm{Sr}_{2} \mathrm{TiO}_{4}$ a transition occurs to the layered Ruddlesden-Popper structure with octahedral titanium coordination.

In this work, we report for the first time the crystal structure of the high-temperature $\alpha$-phase of $\mathrm{Sr}_{2} \mathrm{TiO}_{4}$. We elucidate the structural differences between the related compounds and discuss possible mechanism driving the structural transition.

[1] Drys, M., Trzebiatowski, W. (1957). Roczniki Chemii. 31, 489.

[2] Gunter, J., Jameson, G. (1984). Acta Cryst. C40, 207.

Keywords: strontium titanate; strontium orthotitanate; tetrahedral titanium coordination; reconstructive phase transition 